\title{
Index
}

Accurette device 23

Adenosarcoma, differential diagnosis 80,81

Arias-Stella reaction 65

Atypical endocervical cells 8

Atypical endometrial cells 8

Atypical glandular cells (AGC) 8,9

Atypical glandular cells of undetermined significance (AGUS) 7,8

Atypical squamous cells (ASC) 8

Autopath 30020

The Bethesda System (TBS), glandular cytology 7-9

Breast cancer, tamoxifen and endometrial cancer risks/management 3-5

Carcinosarcoma

clinical features 77

cytopathology 78,79

diagnosis 77

differential diagnosis $68,80,81$

epidemiology 77

histopathology 77,78

overview 77,78

Cell block preparation 19, 20

Cervicovaginal smear, see Pap smear

Choriocarcinoma, differential diagnosis 68,81

Clear cell endometrial adenocarcinoma (CCEA) cytopathology 61

histopathology 61

Clinical features, endometrial adenocarcinoma 52,53

Combined oral contraceptives (COC), endometrium effects 2,3
Cytoplasm, nonepithelial malignant tumor features 70

Differential diagnosis benign processes $64-66$

choriocarcinoma 68

malignant tumors $66-68$

mesenchymal tumors and carcinosarcoma 68 normal endometrial cells 38,39

Dilatation and curettage (D\&C) 24

Dysfunctional uterine bleeding (DUB), cytology 41

Endobrush device 15

Endocervical adenocarcinoma, differential diagnosis 66

Endocyte device 18

Endometrial aspiration, overview 13

Endometrial brushing techniques (EBT)

cytology assessment 12

effectiveness in endometrial cancer detection

11,12

Endobrush device 15

Endocyte device 18

endometrioid adenocarcinoma diagnosis

$$
56-58
$$

Endopap device 15, 16

Medhosa cannula 13, 14

Mi-Mark endometrial sampling kit 14, 15

overview 13

sampling 24

Spanish experience 16,17

Tao brush 17, 18

Endometrial cells, types 7,32, 33

Endometrial glands, histology 33 
Endometrial hyperplasia (EH), see also Endometrial intraepithelial neoplasia

histopathology

typing criteria 44

WHO classification 44-46

hysteroscopy 30

ultrasound 27

Endometrial intraepithelial neoplasia (EIN)

architectural features 47

contemporary approach to endometrial hyperplasia

46,47

nomenclature 47

Endometrial polyp

cytopathology

classification

Jiménez-Ayala classification $49-51$

prospects 48

practical considerations 49

Spanish Society of Cytology nomenclature 47,48

differential diagnosis 64

epidemiology 42,43

tamoxifen therapy risks $3,42,43$

ultrasound 27

Endometrial stromal sarcoma (ESS)

clinical features 71

cytopathology 71

differential diagnosis $68,72-74$

histopathology 71

low-grade 72

undifferentiated 72

Endometritis

acute versus chronic 40

differential diagnosis 64

fungal infection 41

parasitic infection 41

tuberculosis 40,41

viral infection 41

Endopap device 15, 16

Endoscan device 18

Endosearch device 18

Epidemiology, endometrial cancer

high-risk populations 5,6

incidence trends 1

nonepithelial malignant tumors 69

risk factors

age 1

diet 1

geographic factors 1 hormone therapy 2, 3

human papillomavirus 2

occupational exposure 1

race 1

tamoxifen 3,4

Epithelial cells, endometrium 33, 34

Explora curette 23

Extrauterine adenocarcinoma, differential diagnosis 66,67

Follicular cervicitis, differential diagnosis 73

Grading, endometrioid adenocarcinoma 53-55

Gynosampler 22

High-grade carcinoma 54, 55

Histiocytes, Pap smear 10

Hormone replacement therapy (HRT) benign endometrial cells in Pap smear 10

endometrium effects 2

Human papillomavirus (HPV), endometrial cancer risks 2

Hysteroscopy, office technique 29-31

Hysteroscopy and curettage 25

Immunohistochemistry, endometrioid adenocarcinoma 54

Inflammation, see Endometritis

Intrauterine device (IUD)

benign endometrial cells in Pap smear 10

differential diagnosis of effects 65

Isaac endometrial cell sampler 13

Jiménez-Ayala classification

endometrial hyperplasia 49

endometrioid neoplasia 49-51

Kaposi's sarcoma, differential diagnosis 73,74

Leiomyoma, differential diagnosis 76

Leiomyosarcoma

clinical features 74

cytopathology 74,75

differential diagnosis $68,75,76$

histopathology 74

Liquid-based cytology (LBP)

endometrioid adenocarcinoma diagnosis 58

epithelial cells 34

overview 18,19 
Low-grade carcinoma 54

Lymphoma, differential diagnosis 73

Malignant mesodermal mixed tumors (MMMT)

carcinosarcoma 76-79

differential diagnosis $75,80,81$

recurrences and metastases 79

Masterson Endometrial Biopsy System 23

Medhosa cannula 13, 14

Melanoma, differential diagnosis 75

Menopause, endometrial smear findings

cytology 37

histology 37

Menstrual cycle

menstrual phase

cytology 36

histology 36

proliferative phase

cytology 35

histology 35

secretory phase

cytology 36

histology 36

Mesenchymal tumors, see also Endometrial stromal

sarcoma; Leiomyosarcoma

cytology 59,70

differential diagnosis $68,72-76$

mixed epithelial and mesenchymal tumors

76-81

Microglandular hyperplasia of the cervix 65, 66

Mi-Mark endometrial sampling kit 14, 15

Mixed adenocarcinoma 61

Mucinous adenocarcinoma (MA)

cytopathology 59,60

histopathology 59

Novak curette 22

Nuclei, nonepithelial malignant tumor features 70

Oral contraceptives, see Combined oral contraceptives

Pap smear

benign endometrial cells 9,10

The Bethesda System 7-9

effectiveness in endometrial cancer detection

$11,12,52$

endometrioid adenocarcinoma diagnosis 55, 56

histiocytes 10

psammoma bodies 11

tamoxifen patient monitoring 5
Pathogenesis, endometrial cancer 2

Pipelle de Cornier 22

Prevention, endometrial cancer 6

Primary squamous cell carcinoma of the endometrium (PSCCE)

cytopathology 62,63

histopathology 62

overview 61,62

Psammoma bodies, Pap smear 11

Radiation, endometrial effects 42

Reactive endocervical cells 65

Risk factors, see Epidemiology, endometrial cancer

Sampling techniques, endometrial histopathology

brushing technique 24

dilatation and curettage 24

hysteroscopy and curettage 25

vacuum-based sampling

internal piston devices 22

overview 21

vacuum pump devices 23,24

vacuum syringe 22,23

Screening, endometrial cancer 6

Secondary epithelial tumors, differential diagnosis 68

Serous endometrial adenocarcinoma (SEA)

cytopathology 61

histopathology 60,61

Shedding, nonepithelial malignant tumors 69,70

Small cell carcinoma

cytopathology 63

histopathology 63

Softcyte device 18

Squamous cell carcinoma, differential diagnosis 67,75

Squamous differentiation variant, endometrioid adenocarcinoma 58, 59

Stroma, histology 33, 34

Tamoxifen, endometrial cancer risks and management $3-5$

Tis-U-Trap device 23

Transvaginal ultrasound, see Ultrasound

Tuberculosis, endometritis 40, 41

Ultrasound

Doppler ultrasound 28, 29

endometrial sonostructure 27 
Ultrasound (cont.)

endometrial thickness 27

hysterosonography 28

3D ultrasound 29

vaginal ultrasound uses 26, 27

Undifferentiated carcinoma 63

Vabra aspirator 23

Villoglandular variant, endometrioid adenocarcinoma 59

\section{WHO classification}

endometrial hyperplasia 44-46

uterine corpus tumors 69

Z-sampler 22 
